\title{
Viroid RNA systemic spread may depend on the interaction of a 71-nucleotide bulged hairpin with the host protein VirP1
}

\author{
ELSA MANIATAKI, ${ }^{1,2,3}$ MARTIN TABLER, ${ }^{2}$ and MINA TSAGRIS ${ }^{1,2}$ \\ ${ }^{1}$ Department of Biology, University of Crete, Greece, GR-71110 Heraklion/Crete, Greece \\ ${ }^{2}$ Institute of Molecular Biology and Biotechnology, Foundation for Research and Technology, GR-71110 Heraklion/Crete, Greece
}

\begin{abstract}
Viroids are noncoding circular single-stranded RNAs that are propagated systemically in plants. VirP1 is a protein from tomato, which is an excellent host for potato spindle tuber viroid (PSTVd), and it has been isolated by virtue of its specific in vitro binding to PSTVd RNA. We report on the specific in vivo interaction of VirP1 with full-length viroid RNA as well as with subfragments in the three-hybrid system. The terminal right domain (TR) of PSTVd was identified as a strong interacting partner for VirP1. A weaker partner is provided by a right-hand subfragment of hop stunt viroid (HSVd), a viroid that infects tomato poorly. We present a sequence and structural motif of the VirP1-interacting subfragments. The motif is disturbed in the replicative but nonspreading R+ mutant of the TR. According to our in vivo and in vitro binding assays, the interaction of this mutant with VirP1 is compromised. We propose that the AGG/CCUUC motif bolsters recognition of the TR by VirP1 to achieve access of the viroid to pathways that propagate endogenous RNA systemic signals in plants. Systemic trafficking has been suggested for miRNA precursors, of which the TR, as a stable bulged hairpin $71 \mathrm{nt}$ long, is quite reminiscent.
\end{abstract}

Keywords: PSTVd; HSVd; three-hybrid system; RNA binding proteins

\section{INTRODUCTION}

The smallest, so far, replicating RNA molecules that spread within and between organisms are viroids. More than 20 viroids have been described (Flores et al. 1998; Pelchat et al. 2000), exclusively in plants. Their single-stranded circular genome ranges between 0.24 and $0.4 \mathrm{~kb}$ in size, and it has no protein coding capacity (Diener 1999). Presumably, they depend totally on host factors for their propagation; therefore they might be used as probes to directly dissect the pathways that generate, amplify, and traffic systemic RNA signals in plants. No host factors that interact specifically with the nuclear viroids had been found until recently when the tomato VirP1 protein was identified based on its property of interacting with potato spindle tuber viroid (PSTVd) RNA (Martinez de Alba 2000). VirP1 cDNA clones (accession numbers AJ249592-5) were isolated from an expres-

Reprint requests to: ${ }^{3}$ [Present address] Elsa Maniataki, University of Pennsylvania School of Medicine, Department of Pathology/Neuropathology, 603 Stellar-Chance Labs, 422 Curie Boulevard, Philadelphia, PA 19104-6100, USA; e-mail: maniatak@imbb.forth.gr.

Article and publication are at http://www.rnajournal.org/cgi/doi/ 10.1261/rna.2162203. sion library of Lycopersicon esculentum leaves by applying an RNA-ligand screening protocol (Sagesser et al. 1997) using radiolabeled PSTVd RNA as a ligand (Martinez de Alba 2000). In addition to PSTVd RNA, VirP1 binds in vitro to hop stunt viroid (HSVd) (Martinez de Alba 2000). The amino acid sequence of VirP1 does not contain any known RNA-binding motifs; however, a bromodomain and two nuclear localization signals have been identified (Martinez de Alba 2000).

PSTVd is the most well-characterized viroid. It is localized in the nucleus (Schumacher et al. 1983), where it is thought to replicate via the DNA-dependent RNA polymerase II (Rackwitz et al. 1981; Schindler \& Muhlbach 1992) via the rolling circle mechanism (Branch and Robertson 1984). The partial self-complementarity that occurs along the 359 bases of the PSTVd genome gives rise to the secondary structure of an interrupted double helix with a branch at the left terminus (Gast et al. 1996). The viroid molecule appears under the microscope shaped as a rod (Sanger et al. 1976). Extensive recombination events along the rod, which may have occurred between coreplicating viroid molecules (Keese and Symons 1985), have blurred the trace of viroid phylogenesis. Nevertheless, nonautocatalytic viroids have 
been classified in three subfamilies according to the nucleotide sequence of their central conserved region (CCR); the CCR has evolved to three different types, each one of which is very much conserved (Koltunow and Rezaian 1989; Flores et al. 1998). Most viroids have the PSTVd-type CCR and they belong accordingly to the pospivironae subfamily. Within the pospiviroid genus, were PSTVd occurs (Flores et al. 1998), four additional domains can be identified besides the CCR (Keese and Symons 1985).

In the work presented here, we initially aimed at identifying the viroid domain that is responsible for VirP1 binding. We subsequently spotted the core of the VirP1-binding site on viroid RNA and we accordingly focused on particular preexisting viroid mutants for further VirP1-binding studies. The efficiency of the interaction of those mutants with VirP1 that we established can explain their previously reported infective behavior, which actually points out a direction for the exploration of VirP1 function.

\section{RESULTS}

\section{PSTVd RNA and the tomato VirP1 protein specifically interact in vivo, as determined by the three-hybrid system}

The yeast three-hybrid system (SenGupta et al. 1996) was used to study the interaction of VirP1 protein with PSTVd

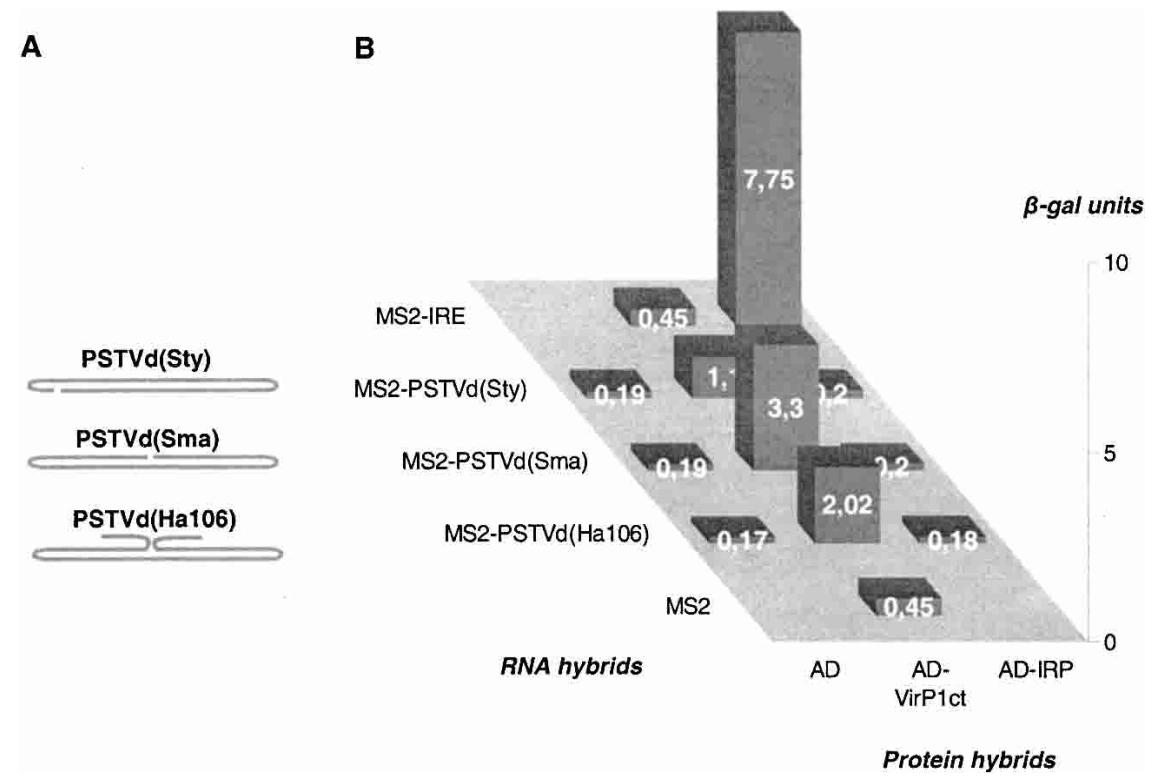

FIGURE 1. Specific interaction between full-length PSTVd RNA and the tomato protein VirP1. (A) The three full-length PSTVd RNAs that were tested in the three-hybrid system for VirP1 binding. The drawings show where PSTVd(Ha106), PSTVd(Sma), and PSTVd(Sty) are linearized. (B) Results of the $\beta$-gal assay. Five RNA hybrids were combined with three protein hybrids, and the respective $S$. cerevisiae strains were tested for $\beta$-gal activity. All five RNA hybrids contain MS2 RNA. Three of them, MS2-PSTVd(Ha106), MS2-PSTVd(Sma), and MS2PSTVd(Sty), contain additionally PSTVd(Ha106), PSTVd(Sma), and PSTVd(Sty) RNA, respectively. MS2-IRE contains the iron responsive element. All the three protein hybrids contain the Gal4 activation domain. AD-VirP1ct additionally contains a 313-amino-acid carboxy-terminal fragment of VirP1. AD-IRP contains the iron responsive protein.
RNA in an in vivo environment. In the three-hybrid system, which is an extension of the two-hybrid system, the study of an RNA-protein interaction is made in Saccharomyces cerevisiae. According to the principle of the three-hybrid system, an RNA hybrid bridges two protein hybrids, and the assembled three-hybrid complex activates transcription of the $\beta$-galactosidase ( $\beta$-gal) gene. The hybrid RNA is a fusion of MS2 RNA with the RNA under study, and it is expressed from an appropriately constructed pIII/MS2-1 vector. The protein under study is fused to the Gal4 trancription activation domain $(\mathrm{AD})$ and this protein hybrid is expressed from an appropriately constructed pACTII vec-

Three different RNA hybrids that contain PSTVd RNA were created: MS2-PSTVd(Ha106), MS2-PSTVd(Sma), and MS2-PSTVd(Sty). The RNA hybrid MS2-PSTVd(Ha106) contains Ha106 PSTVd RNA and was expressed from plasmid construct HaIII/MS2-1. Ha106 is a monomeric PSTVd RNA flanked by repeats of the upper central conserved region (Fig. 1A) and was the RNA ligand used for isolating the VirP1 cDNA clones (Sagesser et al. 1997; Martinez de Alba 2000). The other two RNA hybrids, MS2-PSTVd(Sma) and MS2-PSTVd(Sty), were expressed from plasmids SmaIII/ S2-1 and StyIII/MS2-1, respectively. They contain exact nomers of PSTVd RNA. PSTVd(Sma) RNA represents a viroid linearized at the upper center of the rod, whereas in $\operatorname{PSTVd}($ Sty) RNA, the molecule is linearized at the lower left terminus (Fig. 1A). One protein hybrid with VirP1 was created, ADVirP1ct. It was expressed from plasmid VirP1ctACTII, and it contains the 313amino-acid carboxyterminal part of VirP1. This fragment includes the RNAbinding domain of VirP1 (Martinez de Alba 2000). As a positive control in the three-hybrid test, the interactive pair of hybrids AD-IRP and MS2-IRE was used; iron responsive protein (IRP) interacts with iron responsive element (IRE) RNA (SenGupta et al. 1996). Additionally, with each one of those two hybrids, the specificity of the interaction between VirP1 and PSTVd was tested. The protein-expressing plasmids were combined with the RNA-expressing plasmids in double transformations of the $S$. cerevisiae strain L40-coat. The transformants were subjected to a $\beta$-gal assay that monitors transcriptional activation of the $\beta$-gal gene. The combinations of hybrids and their resulting $\beta$-gal activities are shown in Figure 1B.

The $\beta$-gal units displayed in the second series of Figure $1 \mathrm{~B}$ result from the combination of the RNA hybrid MS2- 
PSTVd(Ha106) with the three protein hybrids AD, ADVirP1ct, and AD-IRP. It is clear that MS2-PSTVd(Ha106) activates $\beta$-gal at higher levels when coexpressed with ADVirP1ct than when coexpressed with AD or with AD-IRP. The result is similar for the other two PSTVd hybrids, MS2PSTVd(Sma) and MS2-PSTVd(Sty), that are displayed at the third and fourth series, respectively. Along the second row of Figure $1 \mathrm{~B}$ it becomes evident that the AD-VirP1ct hybrid does not activate $\beta$-gal significantly when combined with the non-PSTVd containing hybrids, that is MS2 and MS2-IRE. Therefore, the observed $\beta$-gal activation caused by the three PSTVd/VirP1 pairs of hybrids is due to a specific interaction between VirP1 protein and PSTVd RNA in S. cerevisiae.

\section{The terminal right domain of PSTVd binds VirP1}

With the intention of localizing the PSTVd region responsible for VirP1 binding, four overlapping PSTVd subfragments (Fig. 2A) were tested for interaction with VirP1 protein in the three-hybrid system. The rod was initially divided in two pieces of similar length by using the two AvaI restriction sites that lie on PSTVd cDNA at positions 92 and 282 (numbering starts at the left terminus of the circular molecule) resulting in the creation of L170 and R190. L170 is a left-hand subfragment of $0.17 \mathrm{kbp}$ and R190 is a righthand subfragment of $0.19 \mathrm{kbp}$. Similarly, by using EagI (position 144) and AvaII (position 220), two uneven subfragments were created: L280 (Left, $0.28 \mathrm{kbp}$ ) and R80 (Right, $0.08 \mathrm{kbp}$ ). Each PSTVd subfragment is contained in one of the following hybrids, MS2-L170, MS2-R190,

A

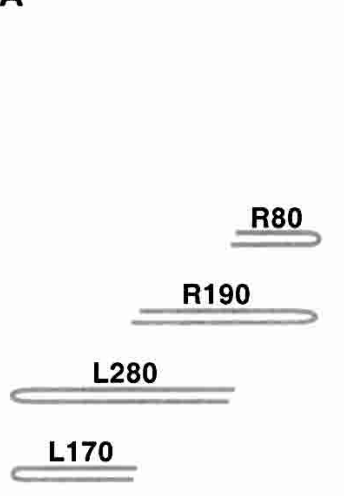

B

MS2-L280, or MS2-R80, which were expressed from plasmids L170III/MS2-1, R190III/MS2-1, L280III/MS2-1, and R80III/MS2-1, respectively.

Each plasmid was used to cotransform the yeast strain L40-coat together with the protein-expressing plasmids as before. The doubly transformed yeast strains were assayed for reporter gene activation, and the results are presented in Figure 2B; MS2-R190 and MS2-R80 highly activate the reporter gene, exclusively when combined with AD-VirP1ct. Activation by MS2-R80 is three times stronger compared to MS2-R190 and two orders of magnitude stronger than MS2-L170 and MS2-L280. The R80 PSTVd subfragment contains the 71-nt TR domain of PSTVd, and our results clearly show that this bulged hairpin is the PSTVd region responsible for VirP1 binding.

\section{A right-hand fragment of HSVd binds VirP1}

VirP1 also interacts in vitro with hop stunt viroid (HSVd; Martinez de Alba 2000). The region of closest similarity between PSTVd and HSVd is the CCR but VirP1 does not bind the CCR of PSTVd (Fig. 2). Therefore, regions outside the CCR of HSVd were examined for their ability to bind VirP1. Toward this end, three HSVd RNA hybrids were created. The first one, MS2-HSV(Sma), contains a fulllength monomeric HSVd RNA linearized at the upper center of the rod by use of the SmaI site (Fig. 3A); it was expressed from plasmid HSVIII/MS2-1. To create the other two RNA hybrids, the HSVd rod was divided in two uneven parts, L190 and R110 (Fig. 3A). The CCR is contained within L190. The hybrids MS2-L190 and MS2-R110 were expressed from plasmids L190III/MS21 and R110III/MS21, respectively. As shown from the $\beta$-gal assay results in Figure 3B, monomeric HSVd RNA interacts with VirP1 in the three-hybrid system. However, the left-hand subfrag-

$\beta-g a l$ units

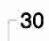

25

20

15

10

5

RNA hybrids

MS2-L170

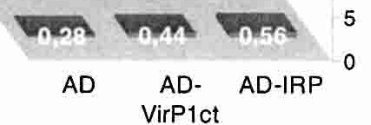

Protein hybrids

FIGURE 2. The TR is responsible for VirP1 binding to PSTVd. (A) The four overlapping PSTVd RNA subfragments that were tested for VirP1 binding in the three-hybrid system. L170 and L280 are left-hand fragments of approximately 170 and 280 bases, respectively. R190 and R80 are right-hand fragments of 190 and 80 bases, respectively. R80 contains the 71-nt TR of PSTVd. (B) Results of the $\beta$-gal assay. The RNA hybrids, MS2-L170, MS2-L280, MS2-R190, and MS2-R80 contain L170, L280, R190, and R80, respectively. They were combined with the protein hybrids $\mathrm{AD}, \mathrm{AD}-\mathrm{VirP} 1 \mathrm{ct}$, and $\mathrm{AD}-\mathrm{IRP}$. ment that contains the CCR does not, as expected. VirP1 is bound to the righthand subfragment R110, which starts where the CCR ends.

\section{The AGG/CCUUC pair of repeats characterizes the PSTVd and HSVd subfragments that interact with VirP1}

The fragmentation of PSTVd (Fig. 2) and HSVd (Fig. 3) disclosed two RNAs, R80 and R110, that interact with the same protein, VirP1. The two sequences were inspected for similarities after being displayed in the rodlike arrangement and as a linear fragment, not considering detailed secondary structure (Fig. 4). 
A

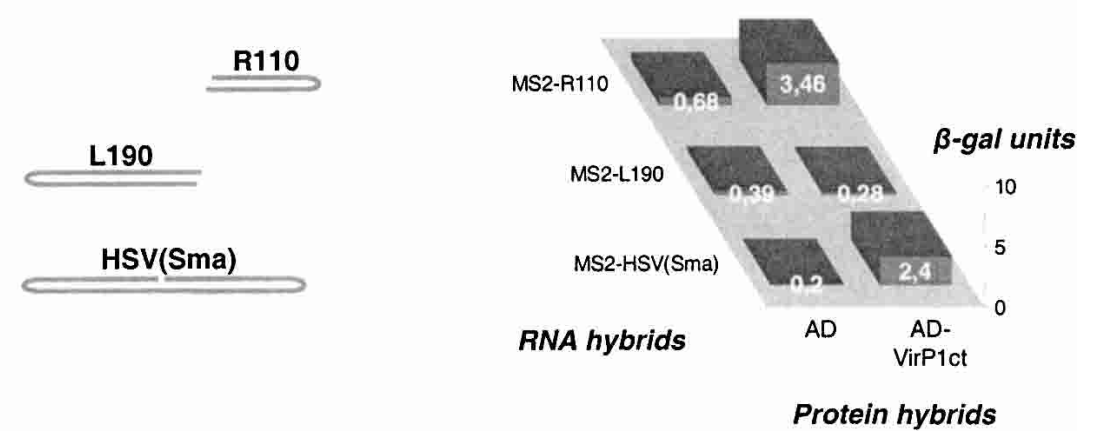

FIGURE 3. VirP1 binds to a right-hand fragment of HSVd. (A) An HSVd RNA monomer, HSV(Sma), and two HSVd RNA subfragments, L190 and R110, were tested. (B) Results of the $\beta$-gal assay. The three RNA hybrids, MS2-HSV(Sma), MS2-L190, and MS2-R110, were combined with the protein hybrids $\mathrm{AD}$ and $\mathrm{AD}-\mathrm{VirP} 1 \mathrm{ct}$.

The major common sequence element between R80 and R110 is the pentanucleotide CCUUC. It is present repeatedly at the bottom strand of these two subfragments, and is embedded in a pyrimidine-rich context that is also repeated within each RNA but is different between the two. Actually, at the bottom strand of R80 the heptanucleotide CCUUCCU is repeated twice whereas at the bottom strand of R110 the hexanucleotide CCUUCU is repeated twice. The top strands also contain repeats which, however, are purine rich: In R80 the pentanucleotide ACAGG is repeated twice whereas the pentanucleotide AGAGG is repeated three times in R110. These two pentanucleotides share the trinucleotide AGG. Summarizing, the most prominent common feature between R80 and R110 is the AGG/ CCUUC pair of repeats. Importantly, this feature is not present on the viroid subfragments PSTVd L280 and HSVd L190, which do not bind the VirP1 protein (Figs. 2 and 3). Subsequent VirP1-binding tests (Martinez de Alba 2000) with three additional viroids, citrus exocortis viroid (CEVd), apple scar skin viroid (ASSVd), and grapevine yellow speckle viroid (GYSVd) showed that, as expected, only the one that contains an AGG/CCUUC motif, that is, CEVd, binds VirP1. The strict correlation between the presence of the AGG/CCUUC motif on a viroid RNA sequence and recognition of this RNA by VirP1 suggests that recognition of a viroid molecule by VirP1 depends on the AGG/CCUUC motif.

\section{The PSTVd R+ mutation reduces the interaction with VirP1}

According to our hypothesis that the AGG/CCUUC motif is the core of a VirP1-binding site on viroid RNA, there are two VirP1-binding sites on PSTVd structural identity with PSTV that spans 18 bases, 2 bp away from the terminal right loop (Fig. $5 \mathrm{~A})$. If this region can be an autonomous VirP1-binding site, then the protein will bind to an extreme terminal righthand PSTVd fragment that contains it. To test this, R34 RNA was designed (Fig. 5B). It is a PSTVd-derived semisynthetic RNA of 34 nucleotides; it contains the terminal right loop, the two stems and the bulge in which the motif is included, and additionally a synthetic 3-bp stem (Fig. 5B). R34 can be transcribed in vitro with T7 polymerase from the R34UC18 plasmid after digestion with SmaI or AvaI. In Northwestern experiments, the R34 RNA interacted specifically with bacterially expressed VirP1, both with the fulllength protein and with a carboxyterminal part. The observed binding, which was weaker than that of the fulllength PSTVd RNA, was highly thermolabile (unpubl. results). The R34 RNA was then replaced with the R34Taq RNA. R34Taq was transcribed from an R34UC18 Taq Idigested template. The $\mathrm{Taq}^{a} \mathrm{I}$ restriction site lies downstream from the SmaI restriction site in the pUC18

$$
5^{-}
$$

R80(PSTVd)

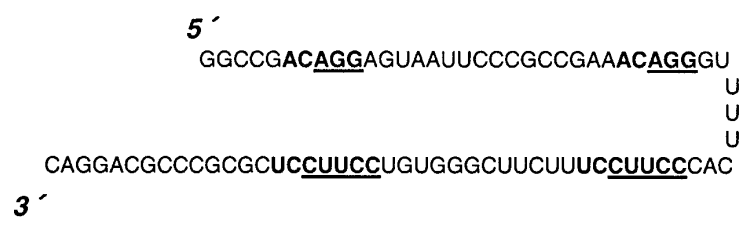

$5^{\circ}$ CAGAAUCCAGCGAGAGGCGUAGGAGAGAGGGCCGCGGUGCUCUGGAGUAGAGG R110(HSVd) UUCUCGGUCCUCUUCCAUUUUCUUCUUCCCUGCUAGCUACCACAAAGCUUCGUC $3^{\prime}$

FIGURE 4. Comparison of the nucleotide sequences of the viroid subfragments R80(PSTVd) and R110(HSVd). Each sequence is shown folding back on itself, as predicted for the interrupted double-helical viroid conformation. The repeats that are observed within each strand of the subfragments are presented in bold characters. The sequences shared by PSTVd and HSVd are underlined. 
A

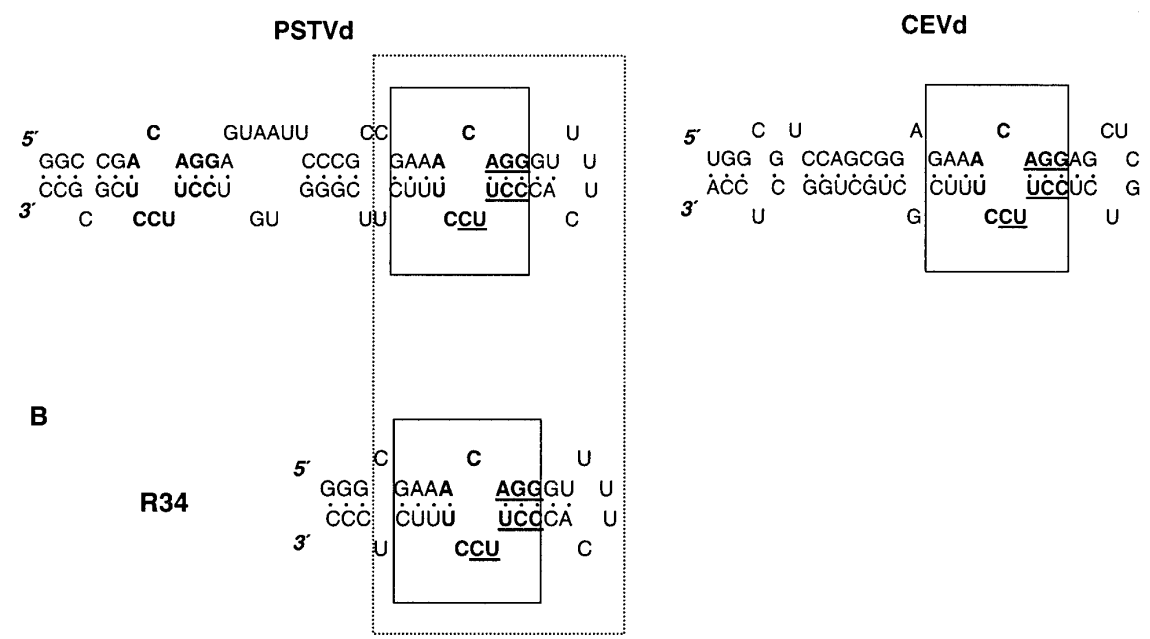

FIGURE 5. (A) Comparison between the terminal right regions of PSTVd and CEVd. Their 18-nt region of sequence and structural identity is boxed (solid line). The repeated ACAGG/ CCUUCCU pair that includes the (underlined) AGG/CCUUC motif is presented in bold characters. $(B)$ The designed R34 RNA. The dashed box indicates the 28-nt part that PSTVd and R34 share.

polylinker; therefore, $14 \mathrm{nt}$ that derive from the pUC18 polylinker are added to the $3^{\prime}$ end of R34. The 14-nt tail can fold into a 4-bp stem. Alternatively, a couple of tails can form a dimer. In any case, the secondary structure of the R34 part is not affected, and the extra "Taq ${ }^{a} I$ RNA" presumably offers unspecific contacts with VirP1 that stabilize binding to R34, which by itself is apparently too short.

In addition to the wild-type PSTVd, two more sequences were created (Fig. 6A); they represent the mutations PSTVd $\mathrm{R}+$ and variant 1 (Hammond 1994). This was done to test the correlation of the VirP1-binding ability with the biological effects of those mutants. PSTVd $\mathrm{R}+$ has a unique phenotype: It replicates in the crown gall of tomato plants that have been injected with agrobacterium that was transformed with PSTVd cDNA, but the only non-wild-type PSTVd RNA that is detected in leaves of some of those plants is PSTVd variant 1 (Hammond 1994). In PSTVd R+, the UU doublet at the upper margin between the terminal stem and the loop is replaced with a GAA triplet. The secondary structure of the AGG/CCUUC motif is affected: The AGG no longer base pairs with CCU, but with CUU, and the UCC bulge has lost the U (Fig. 6A). In variant 1 , the sequence reversions at the upper and lower margins between the stem and the loop result in restoration of the secondary structure of the AGG/CCUUC motif (Fig. 6A). The interaction of the three (WT, R+, var1) RNAs with VirP1 protein was tested in vitro with an electrophoretic mobility shift assay (EMSA) and in vivo with the threehybrid system (Fig. 6B,C).

For EMSA (Fig. 6B), three transcripts, WTR34Taq, $\mathrm{R}+\mathrm{R} 34 \mathrm{Taq}$, and var1R34Taq, were prepared from their templates, the Taq ${ }^{a} \mathrm{I}$ digested plasmids WTR34pUC, $\mathrm{R}+\mathrm{R} 34 \mathrm{pUC}$, and var1R34pUC, respectively. Each one of the three transcripts was incubated with purified his-tagged $\operatorname{VirP} 1_{(290-602)}$. This 313-amino-acid carboxyterminal fragment of VirP1 was expressed from plasmid pHis-VirP1 $1_{(290-602)}$ (Martinez de Alba 2000). Free and VirP1-bound RNAs were separated in a native polyacrylamide gel (Fig. 6B). With no added protein, all three RNAs are resolved in two major bands. With the addition of VirP1, the slower migrating band shifts fully. This is actually true for WT and var1; but in the case of $\mathrm{R}+$ a very low percentage of the RNA is shifted. This shows that the $\mathrm{R}+$ mutation reduces to a great extent the ability of PSTVd RNA to form in vitro complex with VirP1.

The faster migrating band, which is not significantly retarded (Fig. 6B), apparently represents a form that cannot be recognized efficiently by VirP1. The EMSA experiment has been repeated with one modification: All three transcripts were boiled for $2 \mathrm{~min}$ and snap-cooled at $4^{\circ} \mathrm{C}$ before incubation with the protein (Fig. 6B). The slower migrating band in the proteinfree sample disappears, and, as expected, so does the major retarded band in the VirP1-containing sample. Presumably, boiling destroys RNA conformation or dimer formation; therefore, efficient binding to VirP1 can no longer occur.

For the three-hybrid assay (Fig. 6C), the WTR34Taq, $\mathrm{R}+\mathrm{R} 34 \mathrm{Taq}$, and var1R34Taq RNAs were expressed in the $S$. cerevisiae strain L40-coat as part of the RNA hybrids MS2R34WT, MS2-R34R+, and MS2-R34var1, respectively. Those hybrids, which were expressed from plasmids WTR34III/MS2-1, R+R34III/MS2-1, and var1R34III/MS21 , respectively, were combined with the protein hybrid ADVirP1ct. In the presence of AD-VirP1ct, the MS2-R34WT RNA hybrid activates $\beta$-gal one order of magnitude more than the negative control MS2, which contains no PSTVd RNA (Fig. 6C). Activation by MS2-R34R+ is double the one observed in the negative control, whereas MS2-R34var1 is intermediate between MS2-R34WT and MS2-R34R+. The conclusion of both the in vivo and the in vitro binding tests is that the $\mathrm{R}+$ mutation has a pronounced negative effect on the interaction of VirP1 with PSTVd RNA. The interaction is at least partially restored for variant 1 .

\section{DISCUSSION}

\section{The PSTVd-type TR, which characterizes the Solanaceae-hosted viroids, is an optimized VirP1 partner}

In this report, a specific in vivo interaction between the tomato protein VirP1 and PSTVd RNA in the three-hybrid system (SenGupta et al. 1996) has been described; a car- 
A

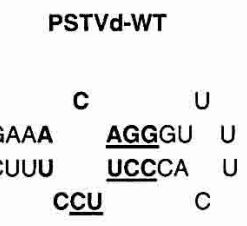

B

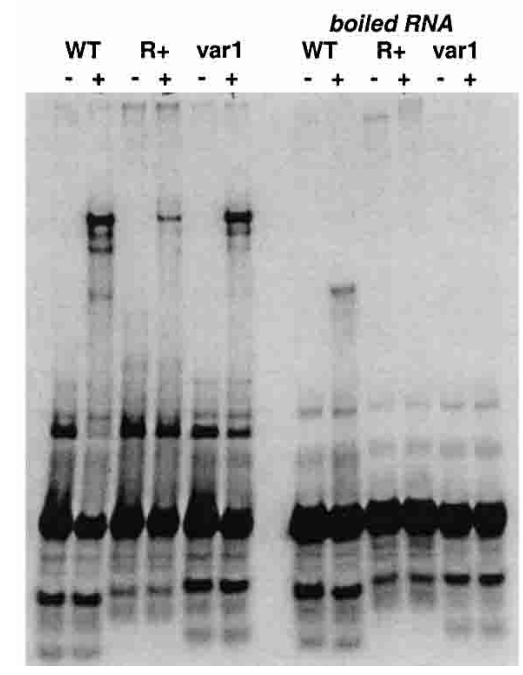

PSTVd-R+

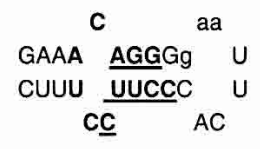

PSTVd-var1

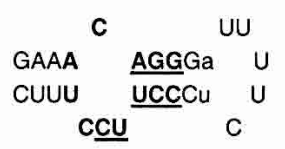

FIGURE 6. The effect PSTVd R+ and PSTVd var1 mutations on the interaction of VirP1 with PSTVd RNA. (A) Primary and secondary structure of the mutated area of PSTVd. The nucleotides that vary from the wild-type sequence are in lower case characters. $(B)$ Electrophoretic mobility shift assay (EMSA). The picture shown here is representative of four such experiments. Radiolabeled WTR34Taq, R+R34Taq, and var1R34Taq RNA transcripts were incubated with $(+)$ and without $(-)$ purified his-tagged VirP1. The experiment is duplicated with boiled and snap-cooled RNA transcripts. $(C)$ The three-hybrid test. The WTR34Taq, R+R34Taq, and var1R34Taq RNAs are contained in the RNA hybrids MS2-R34WT, MS2-R34R+, and MS2R34var1, respectively. Each one of those RNA hybrids, and MS2 as well, were tested with the protein hybrid $\mathrm{AD}$-VirP1ct. In each case, two independent double transformants of the $S$. cerevisiae strain L40-coat were assayed for $\beta$-gal activity.

boxyterminal part of VirP1 interacts with three differently linearized monomeric forms of PSTVd RNA. Activation of $\beta$-gal is increased by at least an order of magnitude when the PSTVd monomer in the RNA hybrid is replaced by R80. R80 represents a structural unit and a functional viroid module, as it contains the 71-nt TR domain of PSTVd, one of the five structural/functional domains of the pospiviroids (Keese and Symons 1985; Flores et al. 1998).

The TR of PSTVd is almost identical to those of tomato planta macho viroid (TPMVd) and tomato apical stunt viroid (TASVd); in fact, among PSTVd, TPMVd, and TASVd, the TR is more conserved than the CCR, and this is also true for chrysanthemum stunt viroid (CSVd; Keese and Symons 1985). It seems that the PSTVd-like TR is optimal, at least for the Solanaceae-hosted viroids, or even for viroids that replicate in plant species belonging to the asterideae grouping. Presumably, the PSTVd-type TR provides a viroid with the VirP1-binding affinity that is required for propagating

in those plants. HSVd does not possess such a strong VirP1-binding region: HSVd R110 activates the reporter gene in the three-hybrid system by an order of magnitude less than PSTVd R80 (Figs. 2, 3). HSVd RNA accumulates slowly and at medium levels in tomato (Sano and Ishiguro 1998); moreover, infectivity varies between HSVd strains (Puchta et al. 1988). Weak VirP1 binding is perhaps what restricts HSVd infectivity in tomato. In this case, an HSVd-PSTVd chimaera that would contain HSVd with a PSTVd-type TR would be more infectious in tomato and other Solanaceae than HSVd. Such a recombinant viroid would evoke columnea latent viroid (CLVd), which possesses a CCR identical to that of HSVd and a TR identical to that of TPMVd (Hammond et al. 1989).

\section{The secondary structrure of the AGG/CCUUC motif in PSTVd and its sequence context maximize VirP1 binding}

Apart from the AGG/CCUUC motif, HSVd R110 and PSTVd R80 do not share other sequence elements (Fig. 4). Furthermore, according to any secondary structrure prediction we tried (unpubl. results), the detailed secondary structure of the motif varies within HSVd and is different from that of PSTVd. Apparently, in PSTVd, VirP1 binding is maximized by the secondary structure of the AGG/CCUUC motifs and the sequence context around. The study of the PSTVd R+ mutation also points to the importance of structure and context for maximal binding to VirP1. PSTVd R+ is mutated in the terminal right loop and, as a result, base pairing at the terminal right stem is altered as if the lower strand has slipped one position rightward; in variant 1 , base pairing is restored back to the wild-type structure (Fig. 6A). Reduced complex formation with VirP1 is observed in the case of PSTVd R+ but not for PSTVd variant 1 (Fig. 6B). Likewise, in the three-hybrid system, the R+ mutation significantly impairs the interaction between PSTVd and VirP1, which is partially restored in variant 1 (Fig. 6C). It is therefore proposed that the tomato VirP1 protein binds RNAs that contain a CCUUC facing an AGG, in a partially duplexed structural context as it happens in HSVd and in PSTVd R+, but binding is maximum when the secondary structure and the sequence context of the AGG/CCUUC motif is that of wild-type PSTVd. 
The left-hand AGG/CCUUC motif region of TR is perhaps a VirP1-binding site not as strong as the extreme right-hand motif region. Even so, two VirP1-binding sites on a viroid molecule are better than one. Noticeably, upon passage of CEVd from tomato, a duplication of the terminal right region of $\mathrm{CEVd}$, which contains one motif (Fig. 5), takes place (Semancik et al. 1993), indicating that there is selection pressure for more than one VirP1-binding sites on a viroid molecule during infection of a tomato plant.

\section{How viroid systemic spread can depend on VirP1}

The findings presented in this report suggest that the contribution of the TR to viroid infectivity may include interaction with VirP1. The functional significance of the interaction between the TR and VirP1 is revealed by the correlation between the VirP1-binding ability and the biological effects of the two PSTVd mutants, $\mathrm{R}+$ and variant 1 . PSTVd $\mathrm{R}+$ is not infectious in tomato by mechanical inoculation; however, it replicates in the crown gall of tomato plants injected with PSTVd-transformed agrobacterium (Hammond 1994). No PSTVd RNA is detected at the leaves of most of those injected tomatoes; only in a few plants is the viroid detected at the leaves, but this RNA is PSTVd wildtype and variant 1 (Hammond 1994). Defective VirP1 binding (Fig. 6) can explain the inability of PSTVd R+ to spread systemically. The restoration, at least partially, of the interaction with VirP1 (Fig. 6) can explain the presence of variant 1 at the leaves. It is therefore suggested that the interaction of PSTVd with VirP1 is required for the systemic spread of the viroid.

Proposing a function for VirP1 depends on how the nonsystemic-spread phenotype of PSTVd $\mathrm{R}+$ is interpreted. This phenotype could simply arise from a defect in the initiation of replication, which in the crown gall is overcome because of the continuous synthesis of a high number of transcripts. VirP1 sequence contains nuclear localization signals and a bromodomain (Martinez de Alba 2000), so the protein perhaps delivers PSTVd to polII in the nucleus. The maize trancription factor KNOTTED1 transports the knotted 1 mRNA through plasmodesmata in the developing mesophyll (Lucas et al. 1995). VirP1 may additionally (or alternatively) contribute to the intercellular transport of PSTVd RNA, which moves through plasmodesmata (Ding et al. 1997) and uses the phloem (Palukaitis 1987), where it can be detected (Stark-Lorenzen et al. 1997) and replicated (Zhu et al. 2001). Perhaps VirP1 allows access of the viroid to pathways that propagate systemically endogenous RNA signals. Recruitment of VirP1 by the TR might then result in suppression of the endogenous function, but this could also contribute to PSTVd propagation. Suppression of PTGS actually might be required for viroid systemic spread, as PSTVd is a target of PTGS (Itaya et al. 2001; Papaefthimiou et al. 2001). The non-systemic-spread phenotype of PSTVd
$\mathrm{R}+$ (Hammond 1994) may then also result from failure of the mutated TR to suppress PTGS. The TR apparently would compete out the endogenous RNA partners of VirP1. Excellent candidates as lower affinity endogenous partners of VirP1 would be the miRNA precursors, because of their remarkable similarity in size and overall secondary structure to the TR. Importantly, it has been suggested (Jorgensen 2002) that miRNA precursors may move systemically, and it has been shown that they are produced in the nucleus (Lee et al. 2002). Does a pathway of internuclear systemic RNA propagation exist? If so, any invaders could at least be useful in tracking it down.

\section{MATERIALS AND METHODS}

\section{Plasmid constructions}

The protein-expressing plasmid VirP1ctACTII was constructed by ligating the $1.4 \mathrm{~kb}$ SmaI-XhoI fragment of plasmid 19III/45 (a gift of Rudolf Sagesser, IMBB, FORTH, Greece) into a SmaI-XhoI doubly digested pACTII plasmid (SenGupta et al. 1996). Each one of the RNA-expressing plasmids that were used in the three-hybrid system contains an insert at the SmaI site of plasmid pIII/MS2-1 (SenGupta et al. 1996). Specifically, HaIII/MS2-1 contains the 0.4$\mathrm{kb}$ EcoRI-HindIII fragment of plasmid pHa106 (Tsagris et al. 1991). StyIII/MS2-1 and SmaIII/MS2-1 contain, respectively, the $0.36-\mathrm{kb}$ Sty I fragment and the 0.36-kb SmaI fragment of plasmid pSP-Av5.8(-) (Tabler and Sanger 1985). L170III/MS2-1 and R190III/MS2-1 contain, respectively, the 0.17-kb AvaI fragment and the $0.19-\mathrm{kb}$ AvaI fragment of plasmid pSP2B(+) (Tsagris et al. 1991). L280III/MS2-1 and R80III/MS2-1 contain, respectively, the 0.28-kb AvaII-EagI fragment and the 0.08-kb EagI-AvaII fragment of plasmid pSP-Av4.2(+) (Tabler and Sanger 1985). HSVIII/MS21, L190III/MS2-1, and R110III/MS2-1 contain, respectively, the 0.3-kb SmaI fragment, the 0.19-kb EarI fragment, and the 0.117$\mathrm{kb}$ EarI fragment of plasmid pHP121 (a gift of Holger Puchta, Institute of Plant Genetics and Crop Plant Research, Gatersleben, Germany). WTR34III/MS2-1, R+R34III/MS2-1, and var1R34III/ MS2-1 contain, respectively, the $0.09-\mathrm{kb} \mathrm{Taq}^{a} \mathrm{I}$ fragments of plasmids WTR34pUC, R+R34pUC, and var1R34pUC. The plasmids WTR34pUC, R+R34pUC, and var1R34pUC were constructed by ligating, into pUC18 doubly digested with SmaI and XbaI, the XT7R34WT, XT7R34R+, and XT7R34var1 double-strand DNAs, digested with XbaI. The XT7R34WT, XT7R34R+, XT7R34var1 double-strand DNAs were prepared by annealing the oligonucleotide XT7 with the respective oligonucleotides, T7R34WT, T7R34R+, or T7R34var1, and extending with a Klenow reaction.

Oligonucleotide sequences:

XT7: GGTCTAGAGTAATACGACTCACTATAGG;

T7R34WT: GGGAGAAAGGAAGGGTGAAAACCCTGTTTCGCC CTATAGTGAGTCGTATTAC;

T7R34R+: GGGAGAAAGGAAGGGTGAATTCCCCTGTTTCGC CCTATAGTGAGTCGTATTAC;

T7R34var1: GGGAGAAAGGAAGGGㅁGAAAATCCCTGTTTCG CCCTATAGTGAGTCGTATTAC. 


\section{Yeast methods}

The yeast strain L40-coat (SenGupta et al. 1996) was cultured and transformed according to standard protocols (Ausubel et al. 1987). In all cases, two independent transformants were assayed, at least twice, for $\beta$-gal activity with ONPG (Ausubel et al. 1987). The $\beta$-gal units were calculated as O.D. ${ }_{420} \times 1.000$ divided by the product O.D. ${ }_{550} \times$ volume $(\mathrm{ml}) \times$ time $(\mathrm{min})$.

\section{Electrophoretic mobility shift assay}

For EMSA, the RNAs were transcribed in vitro in the presence of ${ }^{32}$ P-UTP. About 5000-10,000 cpm of radiolabeled transcript were incubated for $1 \mathrm{~h}$ with $17.7 \mu \mathrm{M} \operatorname{VirP} 1_{(290-602)}$, purified according to Michela Denti (in prep.), in the presence of $1 \mu \mathrm{g} / \mu \mathrm{L}$ tRNA and in binding buffer (10 mM HEPES- $\mathrm{NaOH}$ at $\mathrm{pH} 8.0,50 \mathrm{mM} \mathrm{KCl}$, $0.1 \mathrm{mM}$ EDTA, $5 \%$ glycerol). The reaction volume was $10 \mu \mathrm{L}$. The samples were loaded on a nondenaturing $6 \%$ polyacrylamide gel (29:1, acryl:bisacryl ratio) and subjected to electrophoresis in $0.5 \times$ TBE at a constant voltage of $200 \mathrm{~V}$. The gel was dried and an X-ray film was exposed.

\section{RNA secondary structure predictions}

The MFOLD program (Mathews et al. 1999) was used from the URL http://bioinfo.math.rpi.edu/ mfold/rna/form1.cgi.

\section{ACKNOWLEDGMENTS}

The authors deeply acknowledge Marvin Wickens' lab for the three-hybrid system constituents, Rudolf Sagesser for his VirP1plasmid 19III/45, Ioannis Papaefthimiou for help in plasmid constructions, Emilio Martinez for the EMSA protocol, Mariana Gozmanova for one preparation of purified His-tagged VirP1 protein, and Michela Denti for the suggestion of boiling the RNA. Michela Denti, Mariana Gozmanova, Rose Hammond, Kriton Kalantidis, Emilio Martinez, and Rudolf Sagesser provided valuable comments on the manuscript.

The publication costs of this article were defrayed in part by payment of page charges. This article must therefore be hereby marked "advertisement" in accordance with 18 USC section 1734 solely to indicate this fact.

Received October 16, 2002; accepted December 13, 2002.

\section{REFERENCES}

Ausubel, F.M., Brent, R., Kingston, R.E., Moore, D.D., Seidman, J.G., Smith, J.A., and Struhl, K. (eds.) 1987. Current protocols in molecular biology. John Wiley and Sons, New York, NY.

Branch, A.D. and Robertson, H.D. 1984. A replication cycle for viroids and other small infectious RNA's. Science 223: 450-455.

Diener, T.O. 1999. Viroids and the nature of viroid diseases. Arch. Virol. Suppl. 15: 203-220.

Ding, B., Kwon, M.-O., Hammond, R., and Owens, R. 1997. Cell-tocell movement of Potato Spindle Tuber Viroid. Plant J. 12: 931936.

Flores, R., Randles, J.W., Bar-Joseph, M., and Diener, T.O. 1998. A proposed scheme for viroid classification and nomenclature. Arch. Virol. 143: 623-629.

Gast, F.U., Kempe, D., Spieker, R.L., and Sanger, H.L. 1996. Secondary structure probing of potato spindle tuber viroid (PSTVd) and sequence comparison with other small pathogenic RNA replicons provides evidence for central non-canonical base-pairs, large Arich loops, and a terminal branch. J. Mol. Biol. 262: 652-670.

Hammond, R.W. 1994. Agrobacterium-mediated inoculation of PSTVd cDNAs onto tomato reveals the biological effect of apparently lethal mutations. Virology 201: 36-45.

Hammond, R., Smith, D.R., and Diener, T.O. 1989. Nucleotide sequence and proposed secondary structure of Columnea latent viroid: A natural mosaic of viroid sequences. Nucleic Acids Res. 17: 10083-10094.

Itaya, A., Folimonov, A., Matsuda, Y., Nelson, R.S., and Ding, B. 2001. Potato spindle tuber viroid as inducer of RNA silencing in infected tomato. Mol. Plant Microbe Interact. 14: 1332-1334.

Jorgensen, R.A. 2002. RNA traffics information systemically in plants. Proc. Natl. Acad. Sci. 99: 11561-11563.

Keese, P. and Symons, R.H. 1985. Domains in viroids: Evidence of intermolecular RNA rearrangements and their contribution to viroid evolution. Proc. Natl. Acad. Sci. 82: 4582-4586.

Koltunow, A.M. and Rezaian, M.A. 1989. A scheme for viroid classification. Intervirology 30: 194-201.

Lee, Y., Jeon, K., Lee, J.T., Kim, S., and Kim, V.N. 2002. MicroRNA maturation: Stepwise processing and subcellular localization. EMBO J. 21: 4663-4670.

Lucas, W.J., Bouche-Pillon, S., Jackson, D.P., Nguyen, L., Baker, L., Ding, B., and Hake, S. 1995. Selective trafficking of KNOTTED1 homeodomain protein and its mRNA through plasmodesmata. Science 270: 1980-1983.

Martinez de Alba, A.E. 2000. Isolation and characterisation of viroidbinding proteins. Universidad del Pais Vasco, Bilbao, Spain.

Mathews, D.H., Sabina, J., Zuker, M., and Turner, D.H. 1999. Expanded sequence dependence of thermodynamic parameters improves prediction of RNA secondary structure. J. Mol. Biol. 288: 911-940.

Palukaitis, P. 1987. Potato spindle tuber viroid: Investigation of the long-distance, intra-plant transport route. Virology 158: 239-241.

Papaefthimiou, I., Hamilton, A., Denti, M., Baulcombe, D., Tsagris, M., and Tabler, M. 2001. Replicating potato spindle tuber viroid RNA is accompanied by short RNA fragments that are characteristic of post-transcriptional gene silencing. Nucleic Acids Res. 29: 2395-2400.

Pelchat, M., Deschenes, P., and Perreault, J.P. 2000. The database of the smallest known auto-replicable RNA species: Viroids and viroid-like RNAs. Nucleic Acids Res. 28: 179-180.

Puchta, H., Ramm, K., and Sanger, H.L. 1988. Molecular and biological properties of a cloned and infectious new sequence variant of cucumber pale fruit viroid (CPFV). Nucleic Acids Res. 16: 8171.

Rackwitz, H.R., Rohde, W., and Sanger, H.L. 1981. DNA-dependent RNA polymerase II of plant origin transcribes viroid RNA into full-length copies. Nature 291: 297-301.

Sagesser, R., Martinez, E., Tsagris, M., and Tabler, M. 1997. Detection and isolation of RNA-binding proteins by RNA-ligand screening of a cDNA expression library. Nucleic Acids Res. 25: 3816-3822.

Sanger, H.L., Klotz, G., Riesner, D., Gross, H.J., and Kleinschmidt, A.K. 1976. Viroids are single-stranded covalently closed circular RNA molecules existing as highly base-paired rod-like structures. Proc. Natl. Acad. Sci. 73: 3852-3856.

Sano, T. and Ishiguro, A. 1998. Viability and pathogenicity of intersubgroup viroid chimeras suggest possible involvement of the terminal right region in replication. Virology 240: 238-44.

Schindler, I.-M. and Muhlbach, H.-P. 1992. Involvement of nuclear DNA-dependent RNA polymerases in potato spindle tuber viroid replication: A reevaluation. Plant Science 84: 221-229.

Schumacher, J., Randles, J.W., and Riesner, D. 1983. A two-dimensional electrophoretic technique for the detection of circular viroids and virusoids. Anal. Biochem. 135: 288-295. 
Semancik, J.S., Szychowski, J.A., Rakowski, A.G., and Symons, R.H. 1993. Isolates of citrus exocortis viroid recovered by host and tissue selection. J. Gen. Virol. 74: 2427-2436.

SenGupta, D.J., Zhang, B., Kraemer, B., Pochart, P., Fields, S., and Wickens, M. 1996. A three-hybrid system to detect RNA-protein interactions in vivo. Proc. Natl. Acad. Sci. 93: 8496-8501.

Stark-Lorenzen, P., Guitton, M.C., Werner, R., and Muhlbach, H.P. 1997. Detection and tissue distribution of potato spindle tuber viroid in infected tomato plants by tissue print hybridization. Arch. Virol. 142: 1289-1296.
Tabler, M. and Sanger, H.L. 1985. Infectivity studies on different potato spindle tuber viroid (PSTV) RNAs synthesized in vitro with the SP6 transcription system. EMBO J. 4: 2191-2199.

Tsagris, M., Tabler, M., and Sanger, H.L. 1991. Ribonuclease T1 generates circular RNA molecules from viroid-specific RNA transcripts by cleavage and intramolecular ligation. Nucleic Acids Res. 19: $1605-1612$.

Zhu, Y., Green, L., Woo, Y.M., Owens, R., and Ding, B. 2001. Cellular basis of potato spindle tuber viroid systemic movement. Virology 279: 69-77. 
RNA 9: 346-354 (2003)

\title{
Viroid RNA systemic spread may depend on the interaction of a 71-nucleotide bulged hairpin with the host protein VirP1
}

ELSA MANIATAKI, MARTIN TABLER, and MINA TSAGRIS

This article will henceforth be considered co-authored by:

\section{ELSA MANIATAKI, ${ }^{1,2,3}$ ANGEL EMILIO MARTINEZ DE ALBA, ${ }^{1}$ RUDOLF SÄGESSER, ${ }^{1}$ MARTIN TABLER, ${ }^{1}$ AND MINA TSAGRIS ${ }^{1,2}$ \\ ${ }^{1}$ Institute of Molecular Biology and Biotechnology, Foundation of Research and Technology-Hellas, GR-71110 Heraklion, Greece \\ ${ }^{2}$ Department of Biology, University of Crete, GR-71110 Crete, Greece}

\begin{abstract}
Reprint requests to: ${ }^{3}$ [Present address] Elsa Maniataki, University of Pennsylvania, School of Medicine, Department of Pathology/Neuropathology, 603 Stellar-Chance Labs, 422 Curie Boulevard, Philadelphia, PA 19104-6100, USA; e-mail: maniatak@mail.med.upenn.edu.
\end{abstract}

In addition, the phrases describing E. Martinez's and R. Sägesser's contributions in the Acknowledgments section should be deleted. 

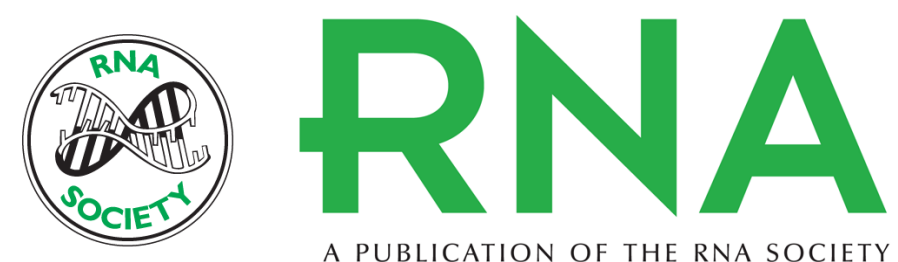

A PUBLICATION OF THE RNA SOCIETY

\title{
Viroid RNA systemic spread may depend on the interaction of a 71-nucleotide bulged hairpin with the host protein VirP1
}

ELSA MANIATAKI, MARTIN TABLER and MINA TSAGRIS

RNA 2003 9: 346-354

\author{
Related Content Viroid RNA systemic spread may depend on the interaction of a 71-nucleotide \\ bulged hairpin with the host protein VirP1 \\ RNA April , 2003 9: 502 \\ References This article cites 30 articles, 7 of which can be accessed free at: \\ http://rnajournal.cshlp.org/content/9/3/346.full.html\#ref-list-1 \\ Articles cited in: \\ http://rnajournal.cshlp.org/content/9/3/346.full.html\#related-urls \\ License \\ Email Alerting $\begin{aligned} & \text { Receive free email alerts when new articles cite this article - sign up in the box at the } \\ & \text { Service }\end{aligned}$ top right corner of the article or click here.
}

